$\underline{\text { Supporting Information }}$

External Electric Field Dependent Photoinduced Charge Transfer in Donor-Acceptor System in Two-Photon Absorption

\author{
Xijiao Mu, ${ }^{\star}$ Huan Zong, ${ }^{\ddagger}$ Lilong Zhu, Mengtao Sun ${ }^{*}$
}

School of Mathematics and Physics, Center for Green Innovation, Beijing Advanced Innovation Center for Materials Genome Engineering, Beijing Key Laboratory for Magneto-Photoelectrical Composite and Interface Science, University of Science and Technology Beijing, Beijing 100083, People's Republic of China.

* Corresponding author. E-mail: mengtaosun@ustb.edu.cn (M. T. Sun)

¿Contribute Equally. 
Table S1. Cartesian coordinate of PC $\mathrm{PC}_{70} \mathrm{BM}$ :PCDTBT compounds optimized by B3LYP/6-31g(d)

\begin{tabular}{|c|c|c|c|c|c|c|c|}
\hline Element & $\mathbf{X}$ & $\mathbf{Y}$ & $\mathbf{Z}$ & Element & $\mathbf{X}$ & $\mathbf{Y}$ & $\mathbf{Z}$ \\
\hline C & -11.7958 & -5.9987 & -2.45561 & $\mathrm{H}$ & -13.9203 & 2.538609 & 1.896345 \\
\hline C & -12.5336 & -7.17024 & -2.29318 & $\mathrm{H}$ & -13.0841 & 3.154495 & 3.313171 \\
\hline C & -12.1142 & -8.1469 & -1.38801 & $\mathrm{C}$ & -15.1185 & 2.531104 & 3.696136 \\
\hline C & -10.9484 & -7.94008 & -0.64823 & $\mathrm{H}$ & -15.81 & 1.747076 & 3.355814 \\
\hline C & -10.2102 & -6.76914 & -0.81286 & $\mathrm{H}$ & -14.9739 & 2.361462 & 4.772735 \\
\hline C & -10.62 & -5.77484 & -1.71815 & $\mathrm{C}$ & -15.7584 & 3.90593 & 3.477612 \\
\hline C & -9.83612 & -4.52486 & -1.89162 & $\mathrm{H}$ & -15.9468 & 4.090299 & 2.412731 \\
\hline C & -9.24301 & -3.90685 & -0.78146 & $\mathrm{H}$ & -16.7155 & 3.991256 & 4.00477 \\
\hline C & -8.5084 & -2.73052 & -0.96541 & $\mathrm{H}$ & -15.1053 & 4.709257 & 3.840532 \\
\hline C & -8.36055 & -2.15936 & -2.25963 & $\mathrm{H}$ & -7.11632 & -1.32912 & 1.781233 \\
\hline C & -8.95521 & -2.78398 & -3.36171 & $\mathrm{C}$ & -0.36389 & 1.32831 & -1.03031 \\
\hline C & -9.68324 & -3.95091 & -3.17527 & $\mathrm{C}$ & 0.41773 & 2.517376 & -1.3164 \\
\hline $\mathbf{N}$ & -7.83953 & -1.93175 & -0.0357 & $\mathrm{C}$ & 0.790088 & 3.124837 & -0.05315 \\
\hline C & -7.26643 & -0.85728 & -0.71806 & $\mathrm{C}$ & 0.239234 & 2.311542 & 1.015618 \\
\hline C & -7.56625 & -0.96742 & -2.1027 & $\mathrm{C}$ & -0.47702 & 1.201969 & 0.411903 \\
\hline C & -6.49616 & 0.198269 & -0.22895 & $\mathrm{C}$ & 1.329224 & 2.509451 & -2.3769 \\
\hline C & -6.024 & 1.170663 & -1.12609 & $\mathrm{C}$ & 2.640944 & 3.100258 & -2.21534 \\
\hline C & -6.32479 & 1.056436 & -2.50596 & $\mathrm{C}$ & 2.996805 & 3.681442 & -1.00597 \\
\hline C & -7.08326 & 0.001189 & -2.99064 & $\mathrm{C}$ & 2.056914 & 3.697201 & 0.094531 \\
\hline C & -5.23029 & 2.292267 & -0.61616 & $\mathrm{C}$ & 2.819908 & 3.473183 & 1.303862 \\
\hline C & -5.27248 & 2.867122 & 0.639353 & $\mathrm{C}$ & 2.292392 & 2.6977 & 2.327591 \\
\hline C & -4.36662 & 3.936518 & 0.801201 & $\mathrm{C}$ & 0.976566 & 2.108897 & 2.184767 \\
\hline C & -3.60389 & 4.209854 & -0.32346 & $\mathrm{C}$ & 1.034769 & 0.794467 & 2.790043 \\
\hline $\mathbf{S}$ & -4.03942 & 3.096465 & -1.613 & $\mathrm{C}$ & 0.34691 & -0.26694 & 2.213273 \\
\hline C & -2.57349 & 5.230405 & -0.452 & $\mathrm{C}$ & -0.42508 & -0.06096 & 1.004393 \\
\hline $\mathbf{C}$ & -2.15082 & 5.99259 & 0.629863 & $\mathrm{C}$ & -0.25224 & -1.24356 & 0.184373 \\
\hline C & -1.1617 & 6.995982 & 0.544059 & $\mathrm{C}$ & -0.13859 & -1.12192 & -1.19563 \\
\hline C & -0.48516 & 7.328912 & -0.62284 & $\mathrm{C}$ & -0.20131 & 0.185061 & -1.81611 \\
\hline C & -0.87616 & 6.562945 & -1.78114 & $\mathrm{C}$ & 0.751269 & 0.180374 & -2.9071 \\
\hline C & -1.90626 & 5.533853 & -1.69087 & $\mathrm{C}$ & 1.49977 & 1.317604 & -3.18061 \\
\hline $\mathbf{N}$ & -0.37326 & 6.688616 & -3.01339 & $\mathrm{C}$ & 0.826869 & -1.92798 & -1.91541 \\
\hline $\mathbf{S}$ & -1.1427 & 5.594589 & -3.96622 & $\mathrm{C}$ & 1.376639 & -1.12596 & -2.96916 \\
\hline $\mathbf{N}$ & -2.14449 & 4.922962 & -2.85357 & $\mathrm{C}$ & 3.605822 & 2.291837 & -2.93721 \\
\hline C & 0.538148 & 8.361644 & -0.66989 & $\mathrm{C}$ & 2.901516 & 1.191679 & -3.52937 \\
\hline $\mathbf{S}$ & 1.049297 & 9.189022 & 0.799699 & $\mathrm{C}$ & 0.601917 & -2.1726 & 0.893534 \\
\hline C & 2.18891 & 10.19471 & -0.07112 & $\mathrm{C}$ & 0.971769 & -1.57037 & 2.142883 \\
\hline C & 2.164758 & 9.878256 & -1.41248 & $\mathrm{C}$ & 2.367931 & 0.589112 & 3.319956 \\
\hline $\mathrm{C}$ & 1.24554 & 8.85585 & -1.75022 & $\mathrm{C}$ & 3.143723 & 1.760707 & 3.034425 \\
\hline C & 3.011589 & 11.19346 & 0.6179 & $\mathrm{C}$ & 4.220029 & 3.345294 & 0.95046 \\
\hline C & 2.599383 & 11.78403 & 1.826921 & $\mathrm{C}$ & 4.331058 & 3.477101 & -0.4734 \\
\hline $\mathrm{C}$ & 3.392084 & 12.73572 & 2.464214 & $\mathrm{C}$ & 5.32135 & 2.767608 & -1.20261 \\
\hline
\end{tabular}




\begin{tabular}{|c|c|c|c|c|c|c|c|}
\hline C & 4.610789 & 13.12644 & 1.906523 & $\mathrm{C}$ & 4.944343 & 2.151149 & -2.4845 \\
\hline C & 5.03253 & 12.54957 & 0.706152 & $\mathrm{C}$ & 3.515877 & -0.0805 & -3.68378 \\
\hline C & 4.247443 & 11.5906 & 0.071935 & $\mathrm{C}$ & 2.725379 & -1.28441 & -3.38557 \\
\hline C & -7.73972 & -2.15127 & 1.415335 & $\mathrm{C}$ & 1.611227 & -2.91019 & -1.25249 \\
\hline C & -6.9958 & -3.45649 & 1.76656 & $\mathrm{C}$ & 1.49564 & -3.03117 & 0.200231 \\
\hline C & -5.59413 & -3.56246 & 1.151787 & $\mathrm{C}$ & 2.964373 & -0.70081 & 3.342174 \\
\hline C & -4.83235 & -4.81839 & 1.595167 & $\mathrm{C}$ & 2.241312 & -1.81371 & 2.729157 \\
\hline C & -3.44676 & -4.94385 & 0.947709 & $\mathrm{C}$ & 5.095851 & 2.500668 & 1.684383 \\
\hline C & -2.66311 & -6.17984 & 1.407659 & $\mathrm{C}$ & 4.535337 & 1.671676 & 2.762863 \\
\hline C & -1.28552 & -6.30967 & 0.744958 & $\mathrm{C}$ & 6.352719 & 2.160185 & -0.4346 \\
\hline C & -0.48555 & -7.52829 & 1.222636 & $\mathrm{C}$ & 6.23959 & 2.027353 & 0.989163 \\
\hline C & 0.889188 & -7.64665 & 0.556077 & $\mathrm{C}$ & 4.921402 & -0.12619 & -3.48899 \\
\hline $\mathbf{H}$ & -12.1463 & -5.234 & -3.1431 & $\mathrm{C}$ & 5.627104 & 0.974361 & -2.89914 \\
\hline $\mathbf{H}$ & -13.4436 & -7.31613 & -2.86957 & $\mathrm{C}$ & 3.402743 & -2.43939 & -2.90487 \\
\hline $\mathbf{H}$ & -12.6892 & -9.06023 & -1.26127 & $\mathrm{C}$ & 2.855088 & -3.24334 & -1.85346 \\
\hline $\mathbf{H}$ & -10.6058 & -8.69759 & 0.052089 & $\mathrm{C}$ & 5.156927 & 0.425644 & 3.053794 \\
\hline $\mathbf{H}$ & -9.28836 & -6.63305 & -0.25392 & $\mathrm{C}$ & 4.384751 & -0.74664 & 3.341136 \\
\hline $\mathbf{H}$ & -9.38894 & -4.33337 & 0.203102 & $\mathrm{C}$ & 3.000599 & -2.88085 & 2.174537 \\
\hline $\mathbf{H}$ & -8.84157 & -2.36396 & -4.35776 & $\mathrm{C}$ & 2.632251 & -3.4816 & 0.927543 \\
\hline $\mathbf{H}$ & -10.1206 & -4.45245 & -4.033 & $\mathrm{C}$ & 3.828999 & -3.95332 & 0.278688 \\
\hline $\mathbf{H}$ & -6.23011 & 0.270067 & 0.819575 & $\mathrm{C}$ & 3.950888 & -3.806 & -1.08971 \\
\hline $\mathbf{H}$ & -5.97581 & 1.823756 & -3.19019 & $\mathrm{C}$ & 4.844299 & -2.48635 & -2.79833 \\
\hline $\mathbf{H}$ & -7.30738 & -0.06552 & -4.05199 & $\mathrm{C}$ & 5.592173 & -1.3509 & -3.09424 \\
\hline $\mathbf{H}$ & -5.96605 & 2.551874 & 1.411321 & $\mathrm{C}$ & 6.73221 & 0.431489 & -2.13621 \\
\hline $\mathbf{H}$ & -4.29356 & 4.507101 & 1.720238 & $\mathrm{C}$ & 7.087491 & 1.0132 & -0.9269 \\
\hline $\mathbf{H}$ & -2.59104 & 5.816803 & 1.605548 & $\mathrm{C}$ & 6.908942 & 0.800377 & 1.378617 \\
\hline $\mathbf{H}$ & -0.92489 & 7.52976 & 1.460012 & $\mathrm{C}$ & 6.370859 & 0.007798 & 2.387932 \\
\hline $\mathbf{H}$ & 2.774192 & 10.39255 & -2.14759 & $\mathrm{C}$ & 4.430657 & -2.9699 & 2.318915 \\
\hline $\mathbf{H}$ & 1.0908 & 8.489425 & -2.75563 & $\mathrm{C}$ & 5.116659 & -1.90455 & 2.866779 \\
\hline $\mathbf{H}$ & 1.640737 & 11.50945 & 2.258627 & $\mathrm{C}$ & 7.446012 & 0.178383 & 0.200618 \\
\hline $\mathbf{H}$ & 3.051192 & 13.17989 & 3.395614 & $\mathrm{C}$ & 6.372705 & -1.43436 & 2.275196 \\
\hline $\mathbf{H}$ & 5.227261 & 13.87061 & 2.40305 & $\mathrm{C}$ & 4.970715 & -3.81526 & 1.221205 \\
\hline $\mathbf{H}$ & 5.983808 & 12.83857 & 0.267218 & $\mathrm{C}$ & 5.20714 & -3.34067 & -1.68791 \\
\hline $\mathbf{H}$ & 4.6007 & 11.12535 & -0.84343 & $\mathrm{C}$ & 6.719308 & -1.01062 & -2.27081 \\
\hline $\mathbf{H}$ & -7.59286 & -4.32711 & 1.468995 & $\mathrm{C}$ & 6.311744 & -3.03347 & -0.90295 \\
\hline $\mathbf{H}$ & -6.92068 & -3.50033 & 2.861878 & $\mathrm{C}$ & 7.045986 & -1.82307 & -1.17789 \\
\hline $\mathbf{H}$ & -5.00874 & -2.6709 & 1.420921 & $\mathrm{C}$ & 7.415507 & -1.2166 & 0.081756 \\
\hline $\mathbf{H}$ & -5.67488 & -3.55448 & 0.05727 & $\mathrm{C}$ & 6.371935 & -3.30461 & 0.564751 \\
\hline $\mathbf{H}$ & -5.42814 & -5.71095 & 1.352337 & $\mathrm{C}$ & 6.912445 & -2.04234 & 1.15022 \\
\hline $\mathbf{H}$ & -4.72419 & -4.81406 & 2.690164 & $\mathrm{C}$ & 6.243829 & -4.64464 & 1.24959 \\
\hline $\mathbf{H}$ & -2.85896 & -4.04025 & 1.167052 & $\mathrm{C}$ & 6.9148 & -4.83875 & 2.591549 \\
\hline $\mathbf{H}$ & -3.5614 & -4.97118 & -0.14583 & $\mathrm{C}$ & 8.260063 & -4.50434 & 2.789252 \\
\hline $\mathbf{H}$ & -3.25377 & -7.08445 & 1.199101 & $\mathrm{C}$ & 6.209461 & -5.44665 & 3.638957 \\
\hline
\end{tabular}




\begin{tabular}{lrrrrrrr}
\hline $\mathbf{H}$ & -2.5383 & -6.14464 & 2.500378 & $\mathrm{C}$ & 8.886236 & -4.76974 & 4.008567 \\
\hline $\mathbf{H}$ & -0.70236 & -5.39677 & 0.935798 & $\mathrm{H}$ & 8.820481 & -4.03191 & 1.987789 \\
\hline $\mathbf{H}$ & -1.41193 & -6.36422 & -0.34668 & $\mathrm{C}$ & 6.833204 & -5.71229 & 4.857453 \\
\hline $\mathbf{H}$ & -1.06592 & -8.44196 & 1.030118 & $\mathrm{H}$ & 5.163341 & -5.70731 & 3.499055 \\
\hline $\mathbf{H}$ & -0.35971 & -7.47336 & 2.313547 & $\mathrm{C}$ & 8.174768 & -5.37352 & 5.045709 \\
\hline $\mathbf{H}$ & 1.437558 & -8.52319 & 0.920522 & $\mathrm{H}$ & 9.930517 & -4.50256 & 4.145284 \\
\hline $\mathbf{H}$ & 1.503639 & -6.76003 & 0.755931 & $\mathrm{H}$ & 6.270272 & -6.18056 & 5.660434 \\
\hline $\mathbf{H}$ & 0.794202 & -7.7422 & -0.53265 & $\mathrm{H}$ & 8.661245 & -5.57728 & 5.995715 \\
\hline $\mathbf{C}$ & -9.10686 & -2.02373 & 2.12103 & $\mathrm{C}$ & 6.277289 & -5.90323 & 0.368207 \\
\hline $\mathbf{H}$ & -9.78303 & -2.81855 & 1.782741 & $\mathrm{H}$ & 5.622262 & -5.76892 & -0.49794 \\
\hline $\mathbf{H}$ & -8.94091 & -2.1997 & 3.192923 & $\mathrm{H}$ & 5.848924 & -6.72717 & 0.954378 \\
\hline $\mathbf{C}$ & -9.77992 & -0.65992 & 1.923975 & $\mathrm{C}$ & 7.683581 & -6.27996 & -0.11576 \\
\hline $\mathbf{H}$ & -9.09849 & 0.133892 & 2.264844 & $\mathrm{H}$ & 8.116527 & -5.44778 & -0.68333 \\
\hline $\mathbf{H}$ & -9.93978 & -0.48413 & 0.852402 & $\mathrm{H}$ & 8.350006 & -6.44933 & 0.735965 \\
\hline $\mathbf{C}$ & -11.1185 & -0.52633 & 2.662642 & $\mathrm{C}$ & 7.663886 & -7.52991 & -0.99806 \\
\hline $\mathbf{H}$ & -11.803 & -1.31695 & 2.321549 & $\mathrm{H}$ & 7.247314 & -8.39171 & -0.4594 \\
\hline $\mathbf{H}$ & -10.9647 & -0.69998 & 3.738245 & $\mathrm{H}$ & 7.015426 & -7.39013 & -1.87335 \\
\hline $\mathbf{C}$ & -11.7807 & 0.842246 & 2.458221 & $\mathrm{C}$ & 9.043262 & -7.91542 & -1.49796 \\
\hline $\mathbf{H}$ & -11.929 & 1.013652 & 1.381927 & $\mathrm{O}$ & 8.980692 & -9.0204 & -2.27575 \\
\hline $\mathbf{H}$ & -11.0929 & 1.631089 & 2.79766 & $\mathrm{C}$ & 10.23732 & -9.47098 & -2.80755 \\
\hline $\mathbf{H}$ & -13.1232 & 0.995802 & 3.18494 & $\mathrm{H}$ & 10.00382 & -10.3558 & -3.40057 \\
\hline $\mathbf{H}$ & -13.8131 & 0.209299 & 2.844598 & $\mathrm{H}$ & 10.68987 & -8.69737 & -3.43376 \\
\hline & -12.9765 & 0.825207 & 4.262027 & $\mathrm{H}$ & 10.92977 & -9.7218 & -1.99945 \\
\hline & -13.775 & 2.368201 & 2.973575 & $\mathrm{O}$ & 10.0747 & -7.32971 & -1.24948 \\
\hline & & & & & & & \\
\hline $\mathbf{H}$ & & & & & & \\
\hline
\end{tabular}




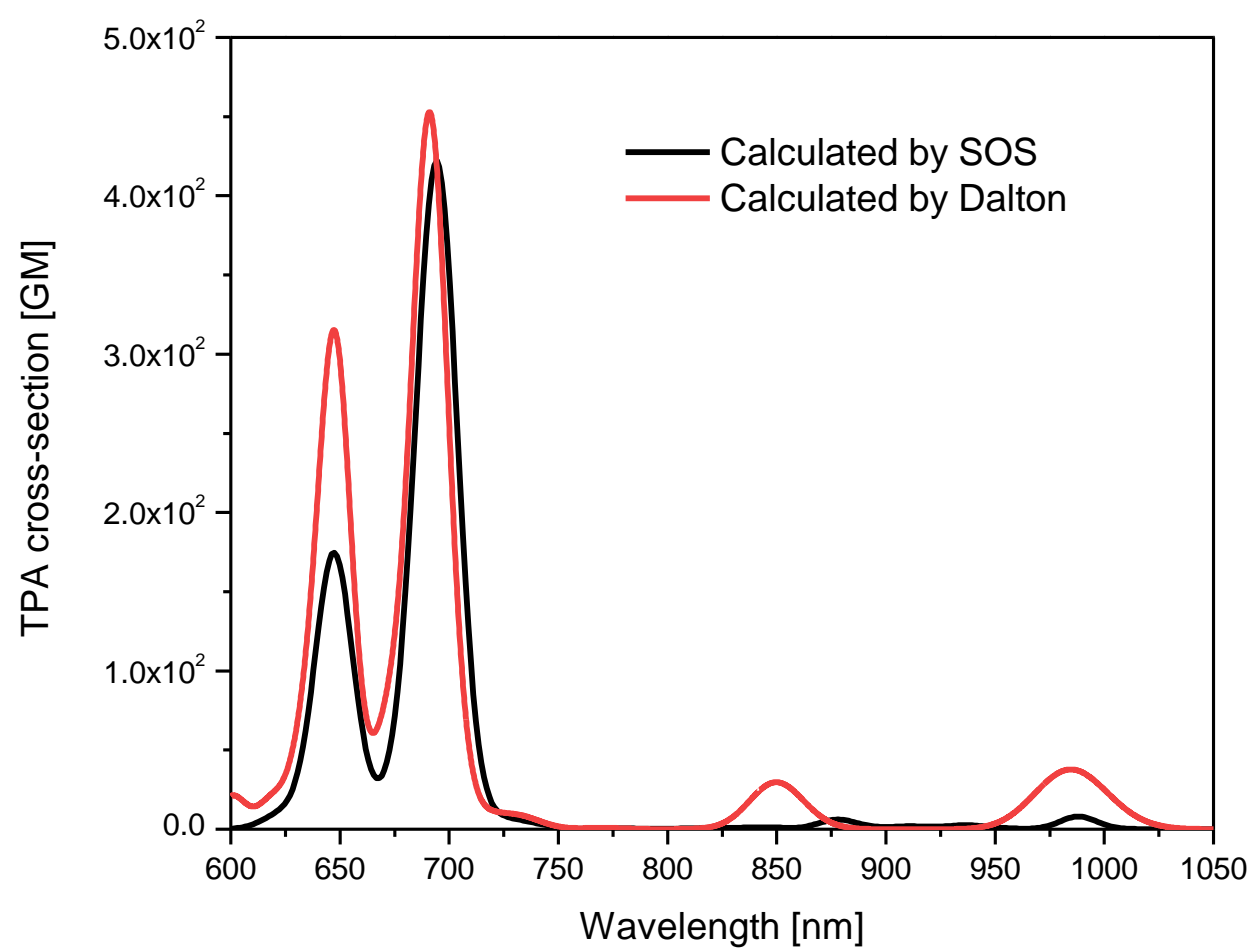

Figure S1. The TPA spectra of $\mathrm{PC}_{70} \mathrm{BM}: \mathrm{PCDTBT}$ without external electric field calculated by SOS method and Dalton-2016. 
(a)

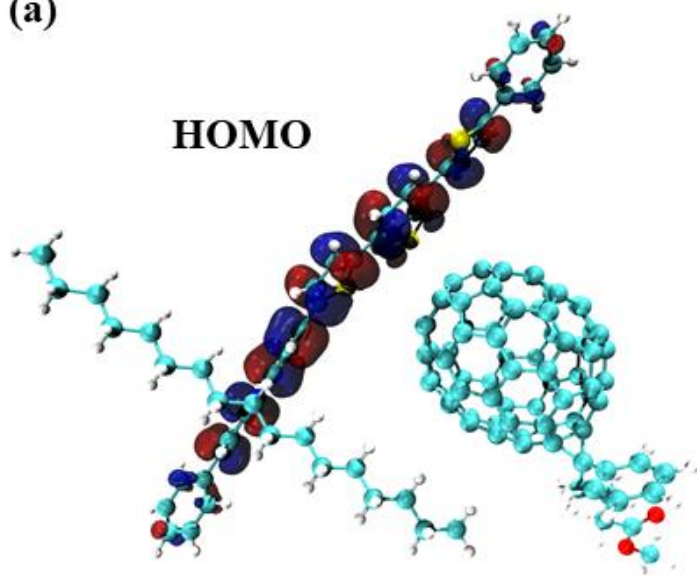

(c)

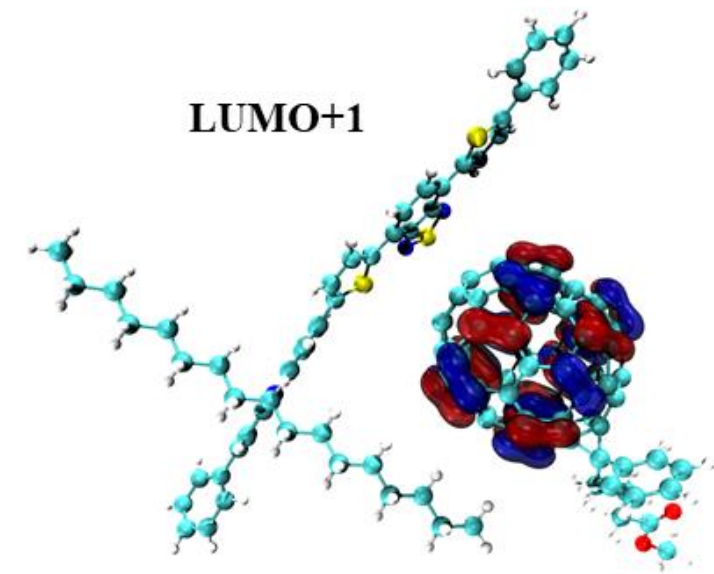

(b)

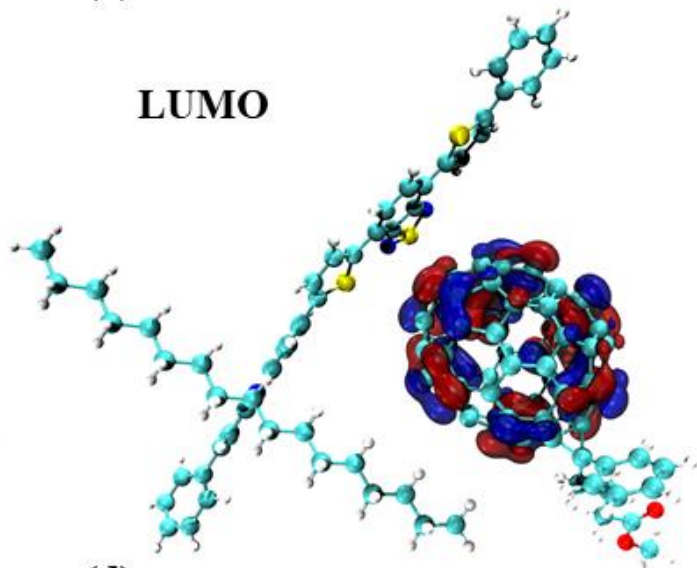

(d)

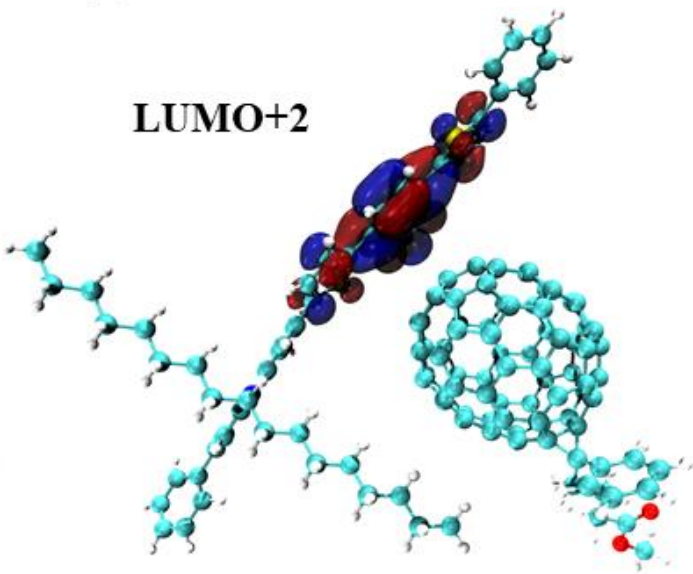

Figure S2. The frontier molecular orbitals, HOMO (a), LUMO (b), LUMO+1 (c) and LUMO+2 (d) of $\mathrm{PC}_{70} \mathrm{BM}: \mathrm{PCDTBT}$.

\section{Complete reference}

[14] Cumpston, B. H. ; Ananthavel, S. P. ; Barlow, S. ; Dyer, D. L. ; Ehrlich, J. E. ;

Erskine, L. L. ; Heikal, A. A. ; Kuebler, S. M. ; Lee, I. Y. S. ; McCord-Maughon, D. ;

Qin, J. ; Röckel, H. ; Rumi, M. ; Wu, X.-L. ; Marder, S. R. ; Perry, J. W.; Two-Photon Polymerization Initiators for Three-Dimensional Optical Data Storage and Microfabrication, Nature, 1999, 398, 51-54.

[22] Yao, H. ; Cui, Y. ; Qian, D. ; Ponseca, C. S. ; Honarfar, A. ; Xu, Y. ; Xin, J. ; Chen, Z. ; Hong, L. ; Gao, B. ; Yu, R. ; Zu, Y. ; Ma, W. ; Chabera, P. ; Pullerits, T. ; Yartsev, A. ;Gao, F. „Hou, J.; 14.7\% Efficiency Organic Photovoltaic Cells Enabled by 
Active Materials with a Large Electrostatic Potential Difference. J. Am. Chem. Soc. 2019, $141,7743-7750$.

[28] Frisch, M. J. ; Trucks, G. W. ; Schlegel, H. B. ; Scuseria, G. E. ; Robb, M. A. ;

Cheeseman, J. R. ;Scalmani, G. ;Barone, V. ;Petersson, G. A. ;Nakatsuji, H. ;Li, X. ; Caricato, M. ; Marenich, A. V. ; Bloino, J. ; Janesko, B. G. ; Gomperts, R. ; Mennucci, B. ; Hratchian, H. P. ; Ortiz, J. V. ; Izmaylov, A. F. ; Sonnenberg, J. L. ; Williams ; Ding, F. ;Lipparini, F. ;Egidi, F. ;Goings, J. ;Peng, B. ;Petrone, A. ;Henderson, T. ; Ranasinghe, D. ;Zakrzewski, V. G. ;Gao, J. ;Rega, N. ;Zheng, G. ;Liang, W. ;Hada, M. ; Ehara, M. ; Toyota, K. ; Fukuda, R. ; Hasegawa, J. ; Ishida, M. ; Nakajima, T. ; Honda, Y. ; Kitao, O. ; Nakai, H. ; Vreven, T. ; Throssell, K. ; Montgomery Jr., J. A. ; Peralta, J. E. ; Ogliaro, F. ; Bearpark, M. J. ; Heyd, J. J. ; Brothers, E. N. ; Kudin, K. N. ;Staroverov, V. N. ;Keith, T. A. ;Kobayashi, R. ;Normand, J. ;Raghavachari, K. ; Rendell, A. P. ; Burant, J. C. ; Iyengar, S. S. ; Tomasi, J. ; Cossi, M. ; Millam, J. M. ; Klene, M. ;Adamo, C. ;Cammi, R. ;Ochterski, J. W. ;Martin, R. L. ;Morokuma, K. ; Farkas, O. ; Foresman, J. B. ; Fox, D. J.; Gaussian 09, 2009. Wallingford CT.

[32] Aidas, K. ; Angeli, C. ; Bak, K. L. ; Bakken, V. ; Bast, R. ; Boman, L. ; Christiansen, O. „Cimiraglia, R. ;Coriani, S. ;Dahle, P. ;Dalskov, E. K. ;Ekström, U. ; Enevoldsen, T. ;Eriksen, J. J. ;Ettenhuber, P. ;Fernández, B. ;Ferrighi, L. ;Fliegl, H. ; Frediani, L. ; Hald, K. ; Halkier, A. ; Hättig, C. ; Heiberg, H. ; Helgaker, T. ; Hennum, A. C. ;Hettema, H. ;Hjertenæs, E. ;Høst, S. ;Høyvik, I.-M. ;Iozzi, M. F. ;Jansík, B. ; Jensen, H. J. A. ; Jonsson, D. ; Jørgensen, P. ; Kauczor, J. ; Kirpekar, S. ; Kjærgaard, T. ; Klopper, W. ; Knecht, S. ; Kobayashi, R. ; Koch, H. ; Kongsted, J. ; Krapp, A. ; 
Kristensen, K. ; Ligabue, A. ; Lutnæs, O. B. ; Melo, J. I. ; Mikkelsen, K. V. ; Myhre, R. H. ;Neiss, C. ;Nielsen, C. B. ;Norman, P. ;Olsen, J. ;Olsen, J. M. H. ;Osted, A. ; Packer, M. J. ; Pawlowski, F. ;Pedersen, T. B. ;Provasi, P. F. ; Reine, S. ; Rinkevicius, Z. ; Ruden, T. A. ; Ruud, K. ; Rybkin, V. V. ; Sałek, P. ; Samson, C. C. M. ; de Merás, A. S. ;Saue, T. ;Sauer, S. P. A. ;Schimmelpfennig, B. ;Sneskov, K. ;Steindal, A. H. ; Sylvester-Hvid, K. O. ; Taylor, P. R. ; Teale, A. M. ; Tellgren, E. I. ; Tew, D. P. ; Thorvaldsen, A. J. ; Thøgersen, L. ; Vahtras, O. ; Watson, M. A. ; Wilson, D. J. D. ; Ziolkowski, M. ;Ågren, H.; The Dalton Quantum Chemistry Program System, WIREs. Comput. Mol. Sci., 2014, 4, 269-284. 